\title{
THE ABDOMINAL PAIN SYNDROME - A STARTING POINT IN DIAGNOSING KIDNEY AND URINARY TRACT DISORDERS IN CHILDREN
}

\author{
Ionela Tămășan ${ }^{1,2}$, Sonia Tănăsescu ${ }^{1,2}$, Liviu Pop ${ }^{1,2}$
}

\begin{abstract}
The acute abdominal pain in children most often represents the reason why they require specialised medical attendance; this pain may be caused by a series of surgical or non-surgical disorders.

The most frequent causes are the ones related to a gastrointestinal disease (mostly gastroenteritis). Nevertheless, one should not neglect the surgical causes, as acute appendicitis remains a condition that must be ruled out in any patient complaining of abdominal pain.

Some of the frequent causes for abdominal pain in children are represented by the urinary tract diseases. Whether we are talking about congenital causes (i.e. birth defects), or about acquired conditions (urinary tract infections, urinary calculi), this type of diseases must be considered when investigating an abdominal pain syndrome.

The study was carried out at the 2nd Pediatrics Clinic within the "Pius Branzeu" County Hospital from Timisoara, on a 2-year interval (2018 and 2019) and it is a retrospective study.
\end{abstract}

The purpose of this paper is to identify the kidney and urinary tract diseases, starting from the acute abdominal pain syndrome as the main symptom. It should be pointed out that our main purpose was to identify the relation between the congenital anomalies in the kidney and the urinary tract infections.

Between January 2018 and December 2019, a number of 3,126 children were admitted to the 2 nd Pediatrics Clinic. Out of these, we have selected only those cases where the abdominal pain could be linked to a kidney or urinary tract disease. Thus, we identified 146 patients where the acute abdominal pain was suggestive of a possible disorder in the urinary tract. Out of the 146 patients, $82(56.16 \%)$ were diagnosed with urinary tract infections. After complex investigations were performed on these patients, we identified urinary tract anomalies in 32 of the cases.

Starting from symptoms which could normally indicate a gastrointestinal disorder (hence the acute abdominal pain), we managed to diagnose a significant number of patients with kidney and urinary tract disorders.

Keywords: acute abdominal pain, urinary tract infection, child

\section{Introduction}

Along the way, pain was given numerous definitions that attempted at including as much as possible the complex facets of this symptom. Thus, in 1979, the International Association for the Study of Pain (IASP) defined pain as "an unpleasant sensory and emotional experience associated with actual or potential tissue damage or described in terms of such damage" [1].

Diagnosing the acute abdominal pain in children is not always easy. The clinical signs of the abdominal pain vary according to the patient's age, pain location and associated symptoms [2,3]. Most often, abdominal pain is self-limiting, benign and relatively easy to diagnose (e.g. viral enteritis, gastroenteritis, constipation, etc.) [4]. The real "cornerstone" for the clinician is to identify the cases of atypical and potentially lethal illnesses, which need an attentive assessment and immediate treatment (especially in surgical cases, like appendicitis, volvulus, peritonitis, etc.) [5].

Acute abdominal pain is frequently indicated by the patients coming to the emergency rooms (i.e. $5-10 \%$ out of the total number of cases) [6,7].

Some of the most frequent causes for abdominal pain in children are represented by the urinary tract diseases [8]. Whether we are talking about congenital causes (i.e. birth defects), or about acquired conditions (urinary tract infections, urinary calculi), this type of diseases must be considered when investigating an abdominal pain syndrome.

\section{Materials and methods}

The study was carried out at the 2nd Pediatrics Clinic within the "Pius Branzeu" County Hospital from Timisoara, on a 2-year interval (2018 and 2019) and it is a retrospective study.

The purpose of this paper is to identify the kidney and urinary tract diseases starting from the acute abdominal pain syndrome as the main symptom. It should be pointed out that our main purpose was to identify the relation between the congenital anomalies in the kidney and the urinary tract infections.

${ }^{1}$ 2nd Pediatrics Clinic, Emergency Clinical Country Hospital "Pius Brînzeu", Timisoara, Romania

2"Victor Babes" University of Medicine and Pharmacy, Timisoara, Romania

E-mail: tamasan.ionela@umft.ro, tanasescu.sonia@umft.ro, liviupop63@yahoo.com 


\section{Results}

Out of the 3,126 children admitted to the 2nd Pediatrics Clinic between January 2018 and December 2019, a number of 807 (25.81\%) had acute abdominal pain as a symptom.

Out of these, we have selected only those cases where the abdominal pain could be linked to a kidney or urinary tract disease. Thus, we identified 146 patients where the acute abdominal pain was suggestive of a possible disorder in the urinary tract.

We have drawn up distribution curves, based on the age, gender and kidney conditions identified in the subjects.

The gender distribution of the patients from the group included in the study shows a prevalence of males $(56 \%)$, while females account for the rest of $44 \%$ - this confirms the information from the literature.

Starting from the abdominal pain, correlated with other clinical data (fever, altered general health state, trouble urinating), but also with biological data (presence of an inflammatory syndrome, urinalysis showing changes suggestive for a urinary tract infection, positive urine culture), we could diagnose a urinary tract infection (UTI) in $82(56.16 \%)$ out of the 146 patients.

Based on the analysis of clinical data, correlated with the inflammatory syndrome, the location of the urinary tract infection has shown that $47(56 \%)$ patients had lower urinary tract infection, while 35 patients (44\%) were diagnosed with upper urinary tract infections.

We have not identified significant differences with regard to age distribution in subjects or the location of the urinary tract infections (upper or lower).

An increased occurrence of lower urinary tract infection (UTI), i.e. cystitis, could be seen in the 6 to 18 -year old age group (60\%), while the cases of upper UTI (pyelonephritis) were rather evenly distributed in the subjects above 1 month of age. (Fig. 1).

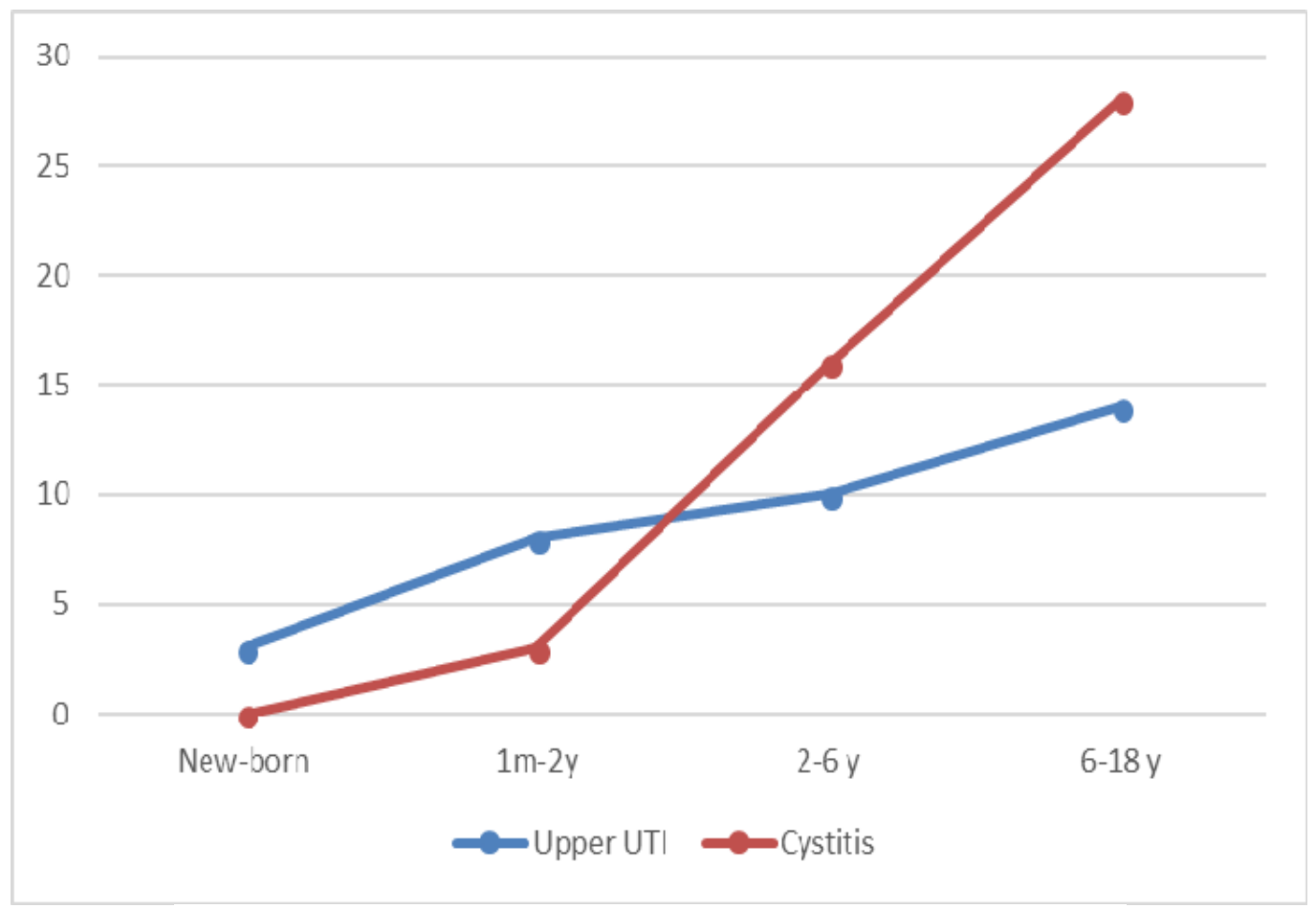

Fig. 1 Distribution of cases according to age and UTI location.

A total number of 195 urine cultures were made for the 146 subjects included in the study. We have included here only the urine cultures performed in order to confirm the diagnosis, and not those urine cultures performed during the regular monitoring, according to protocol, in the patients diagnosed and treated with antibiotics.

Out of the total number of processed urine cultures, $82(42.05 \%)$ tested positive for infection.

The most frequent micro-organisms isolated in the urine cultures of these patients were E. coli - 54 cases (67\%)
[9], followed by Klebsiella - 19 cases (23\%), Proteus - 6 cases $(7 \%)$, and other micro-organisms for the rest of the 3 cases.

As a first conclusion, from the total number of patients included in the study (i.e. 82), E. coli is the most frequent bacteria causing UTI $(p<0.001)$, posing a risk 1.8 times higher than other microorganisms $(R R=1.75)$.

Based on the results from the urine cultures, we can see an increase in the resistance of the E.coli strains to the antibiotics most frequently used to treat children [10]. 
Antibiotic resistance can be seen most often in the case of cephalosporin and aminoglycoside.

Also, we have seen a significant increase in the incidence of Klebsiella strains producing extended-spectrum beta-lactamases (ESBL) [11].

The age distribution in the pediatric patients with infections where Klebsiella BLSE strains were identified has shown that most of the cases were recorded in the 0-24 months age group ( $75 \%$ of the cases), then in the children 2 to 5 years old and 6 to 11 years old, however comparably fewer as the child's age increased.

Out of the 146 patients included in the study, 32 presented congenital anomalies in the kidneys and in the urinary tract system.

After complex assessments were performed on these patients, the following congenital anomalies were found: renal agenesis - 1 case; renal hypoplasia - 2 cases; renal ectopia - 2 cases; horseshoe kidney - 2 cases; cystic diseases - 2 cases; ureteropelvic duplex formation - 5 cases; megaureter - 1 case; hydronephrosis - 10 cases; posterior urethral valve (PUV) disorder - 1 case; abnormality involving the bladder or urethra - 6 cases.

In our study, the male:female ratio related to the congenital anomalies in the kidneys and in the urinary tract system shows a larger prevalence in males [12]. Thus, out of the 32 diagnosed cases, 21 were boys $(65.62 \%)$ and 9 were girls $(34.37 \%)$.

Out of the 32 congenital anomalies, 7 were diagnosed in infants (i.e. the megaureter, 2 cases of ureteropelvic duplex formations, 2 cases of) ureteropelvic junction obstructions, with consecutive hydronephrosis, 2 cases of vesicoureteral reflux). For example, the cases of agenesis/hypoplasia, the cystic kidney disease, the horseshoe kidney were all diagnosed in children above 6 years of age. This should raise some questions, especially if we think of the "extremely technical and computerised" medicine that is available in our century. It is hard to accept that a child can reach 17 or 18 years of age (actually, adult age), without knowing that he/she is missing a kidney, for example.

In every patient, we have assessed the kidney function, by looking at the kidney's capacity to filter the blood and to remove waste products. For this purpose, we have determined the blood urea and creatinine, and later compared the values in their dynamic evolution.

Our study does not include patients with a significant nitrogen retention; however, we had patients who, ever since their congenital anomalies were diagnosed, had a low or "borderline" glomerular filtration rate (GFR).

The results for the serum creatinine were interpreted according to the child's age and based on the reference values. Out of the 32 patients, 28 had normal blood creatinine values, regardless of the time the blood test was performed. One of the patients presented a GFR $<40$ $\mathrm{ml} / \mathrm{min} / 1.73 \mathrm{~m}^{2} \mathrm{SC}$ (also diagnosed with polycystic kidney disease, associated with psychogenic polyuria and mental retardation), while in other 3 cases we have seen blood creatinine above normal values (GFR under $90 \mathrm{ml} / \mathrm{min} / 1.73$ $\mathrm{m}^{2} \mathrm{SC}$ ).

The estimated glomerular filtration rate (eGFR) was calculated using the eGFR calculator, made available by the National Kidney Foundation (an application for Android systems).

Out of the 32 patients diagnosed with congenital anomalies, $19(59.37 \%)$ also presented an urinary tract infection at the moment of diagnosis. In these cases, we have concluded that the acute abdominal pain was caused by the infection and not by the actual congenital anomaly.

Therefore, we can see that the incidence of UTI in children with urinary tract congenital anomalies is statistically larger than in children without such anomalies.

Two of the diagnosed congenital anomalies were most interesting - one, diagnosed in an infant, the other, in a teenager.

\section{Case 1:}

V.A., female, 3 months of age, admitted to the Clinic with loss of appetite, agitation, low-grade fever, excessive crying.

The clinical examination upon hospital admission identified the following: skin lividity, facies with shiners. Optimally fed. No changes in the heart and lungs were identified upon auscultation during the initial visit.

Abdomen sensitive to touch (the child becomes extremely agitated during the examination). External genitals normally developed. Diuresis present. No other changes were identified. $\mathrm{spp} / \mathrm{ml}$ of urine.

Biology tests: Urine culture: > 100,000 Klebsiella

The abdominal ultrasound examination was seen as a natural progression in this case, as it was required both by the symptoms and by the diagnosed urinary tract infection.

The imagery suggest a bilateral grade 2/3 hydronephrosis (more pronounced in the right kidney) Fig.2.

The clinician suspected an ureteropelvic junction obstruction and/or a vesicoureteral reflux (confirmed by higher-quality imaging).

Starting with June 2019, the patient is monitored by the 2nd Pediatric Clinic - the regular visits have shown a favourable evolution, accompanied by other 2 episodes of pyelonephritis.

\section{Case 2:}

C.R., male, aged 14 years, without significant history of illnesses, presents at the emergency room with fever, diarrhea and abdominal pain. The abdominal ultrasound examination does not find the left kidney in the renal fossa. $\mathrm{He}$ is recommended to repeat the ultrasound examination.

The abdominal ultrasound examination identifies the right kidney in the renal fossa, with normal shape and size, without urinary stasis. The left kidney is not found in the renal fossa; a kidney-like structure may possibly exist behind the bladder.

Urinary system MRI investigation - Fig 3: 


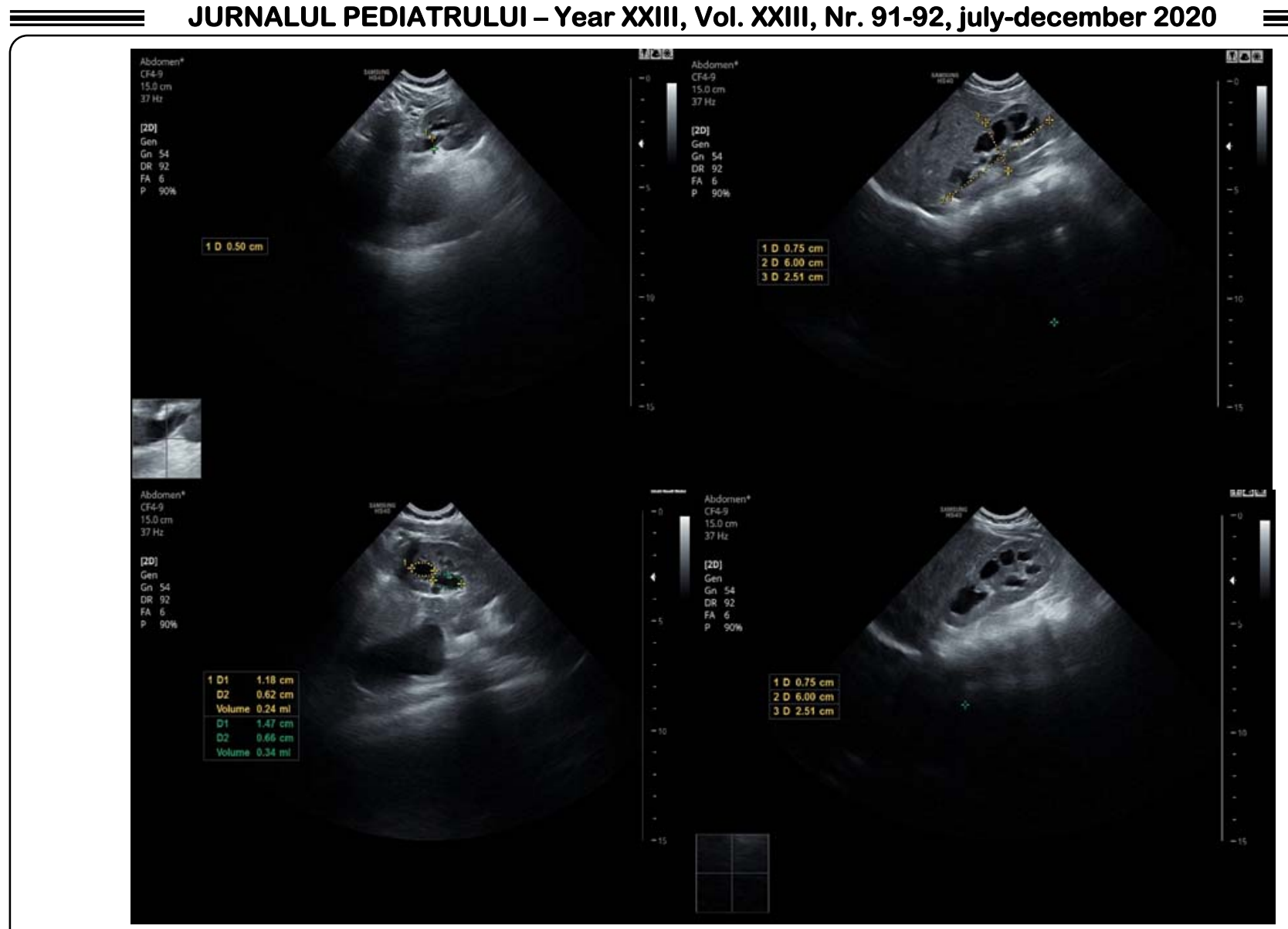

Fig.2 V.A., ultrasound imaging in evolution, with decreased urinary stasis (images from the archive of the Clinic).

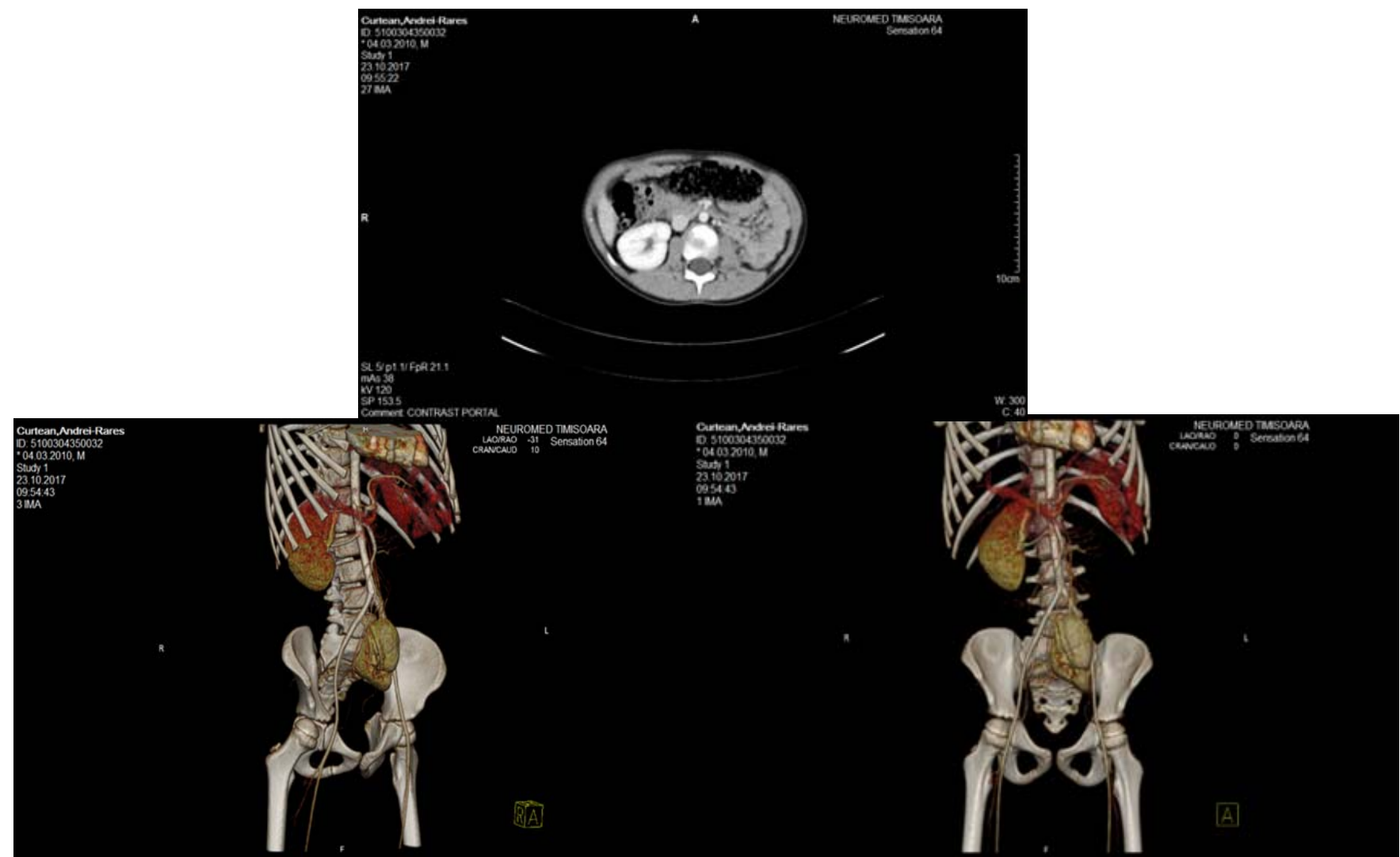

Fig.3 Left ectopic kidney (kidney in the pelvis), diagnosed in a 7-year old child (images from the archive of the 2nd Pediatric Clinic). 


\section{Conclusion}

All the literature reports confirm that the acute abdominal pain is one of the most common pain indicated by children, and also one of the most frequent causes of their presentation at the pediatric emergency rooms [13].

The UTI in the subjects with urinary tract anomalies are caused my strains of microorganisms with increased antibiotic resistance [14]. Regardless of the patient's age, the abdominal ultrasound is the most common method to investigate the urinary tract. The main disadvantage of the ultrasound examination is that it does not provide information on the renal function.
Starting with 2018 and until the present day, the children with kidney and urinary tract anomalies are attentively monitored and regularly assessed. Electronic patient charts are being put in place, to include the clinical, biological and imaging records of these patients, which will ensure an efficient processing of such information.

In conclusion, this paper shows the importance of a crossdisciplinary approach in diagnosing the child patients. Starting from symptoms which could normally indicate a digestive system disorder (hence the acute abdominal pain), we managed to diagnose a significant number of patients with kidney and urinary tract disorders.

\section{References}

1. Sharma KK, Agrawal P, Toshiniwal H. Acquired gastric outlet obstruction during infancy and childhood: a report of five unusual cases. J Pediatr Surg 1997;32:928-930.

2. Ormarsson OT, Haugen SE, Juul I. Gastric outlet obstruction caused by heterotopic pancreas. Eur J Peditr Surg 2003;13:410-413.

3. Azarow K, Kim P, Shandling B, et al. A 45-year experience with surgical treatment of peptic ulcer disease in children. J PediatrSurg 1996;31:750-753.

4. Perdereau S, Touzot F, Robin L, et al: Recurrent pyloric stenosis in a 7-year-old child with chronic granulomatous disease. Arch Pediatr 2013;20(12):13371339.

5. Shukla RM, Mukhopadhyay M, Tripathy BB, et al. Pyloric and antral strictures following corrosive acid ingestion: A report of four cases. J Indian Assoc Pediatr Surg 2010;15(3):108-109.

6. Sharma KK, Ranka P, Goyal P, et al. Gastric outlet obstruction in children: an overview with report of Jodhpur disease and Sharma's classification. J Pediatr Surg 2008;43(10):1891-1897.

7. Chan KL, Saing H. Balloon catheter dilatation of peptic pyloric stenosis in children. J Pediatr Gastroenterol Nutr 1994; 18:465-468.

8. Ratan SK, Dhua AK, Sinha S, et al. Acquired nonspecific cicatrizing inflammation causing pyloric stricture and gastric outlet obstruction in infancy: is it Jodhpur disease? Trop Gastroenterol 2013;34(4):274277.

9. Hameury F, Mcheik J, Lardy H, et al. Acquired non hypertrophic pyloric stenosis in children. Arch Pediatr 2007;14(4):330-333.

\section{Correspondence to:}

Ionela Tămășan,

",Victor Babes"' University of Medicine and Pharmacy

P-ta Eftimie Murgu no. 2, Timisoara, Romania

Phone: 0256/220479

Email: tamasan.ionela@umft.ro 